Supporting Information

\title{
Full Solution-Processed Fabrication of Conductive Hybrid Paper Electrodes for Organic Optoelectronics
}

Yang Yang ${ }^{1}$, Wencong Liu ${ }^{2}$, Quanbo Huang ${ }^{1}$, Xinsheng Li $^{1}$, Hao Ling ${ }^{1}$, Junli Ren ${ }^{1}$, Runcang $\operatorname{Sun}^{3}$, Jianhua Zou ${ }^{2}$, Xiaohui Wang ${ }^{1, *}$

${ }^{\text {I}}$ State Key Laboratory of Pulp and Paper Engineering, South China University of Technology, Guangzhou 510640, China.

${ }^{2}$ Institute of Polymer Optoelectronic Materials and Devices, State Key Laboratory of Luminescent Materials and Devices, South China University of Technology, Guangzhou, 510640, China.

${ }^{3}$ Liaoning Province Key Laboratory of Pulp and Papermaking Engineering, Dalian Polytechnic University, Dalian, 116034, China.

*Corresponding author: Xiaohui Wang

E-mail: fewangxh@scut.edu.cn

Total Number of Pages: 7, Total Number of Figures: 5, Total Number of Tables: 2 
Table S1. The resistances of $\mathrm{Cu}$ NPs/AHP with various experimental parameters.

Table S2. Sheet resistance (Rs) or conductivity of conductive papers based on $\mathrm{Cu}$ NPs or other conductive materials.

Figure S1. Cross-sectional SEM image of the hybrid paper.

Figure S2. The AFM images and the height profiles of $\mathrm{Cu}$ NPs coated on HP with polydopamine as the adhesive layer.

Figure S3. Reflectivity of the Cu NPs/AHP.

Figure S4. Resistance changes of the $\mathrm{Cu}$ NPs/AHP with different bending direction and cycles under curve radius of $10 \mathrm{~mm}(\mathrm{~A})$ and $15 \mathrm{~mm}(\mathrm{~B})$.

Figure S5. SEM and AFM images of hybrid paper before (A) and after acid-etched pretreatment $(\mathrm{B})$.

Table S1. The resistances of $\mathrm{Cu}$ NPs/AHP with various experimental parameters.

\begin{tabular}{|c|c|c|c|}
\hline 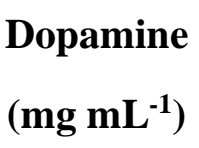 & $\begin{array}{c}\left(\mathrm{NH}_{4}\right)_{2} \mathrm{PdCl}_{4} \\
\left(\mathrm{mg} \mathrm{mL}^{-1}\right)\end{array}$ & $\begin{array}{l}\text { ELD Time } \\
\text { (sec) }\end{array}$ & $\begin{array}{c}\mathbf{R s} \\
\left(\Omega \mathrm{sq}^{-1}\right)\end{array}$ \\
\hline 0.2 & 0.2 & 60 & $1.33 \pm 0.22$ \\
\hline 0.5 & 0.2 & 60 & $1.18 \pm 0.22$ \\
\hline 1.0 & 0.2 & 60 & $1.14 \pm 0.22$ \\
\hline 2.0 & 0.2 & 60 & $1.24 \pm 0.15$ \\
\hline 0.5 & 0.05 & 60 & $16.84 \pm 13.27$ \\
\hline 0.5 & 0.1 & 60 & $3.26 \pm 1.70$ \\
\hline 0.5 & 0.2 & 60 & $1.44 \pm 0.20$ \\
\hline 0.5 & 0.3 & 60 & $1.55 \pm 0.22$ \\
\hline 0.5 & 0.1 & 30 & $66.38 \pm 46.66$ \\
\hline 0.5 & 0.1 & 45 & $4.75 \pm 1.75$ \\
\hline 0.5 & 0.1 & 60 & $3.37 \pm 1.30$ \\
\hline 0.5 & 0.1 & 90 & $1.69 \pm 0.39$ \\
\hline 0.5 & 0.1 & 120 & $1.06 \pm 0.29$ \\
\hline
\end{tabular}


Table S2. Sheet resistance (Rs) or conductivity of conductive papers based on $\mathrm{Cu}$ NPs or other conductive materials.

\begin{tabular}{|c|c|c|c|}
\hline Electrodes & Methods & Rs/conductivity & Ref. \\
\hline $\mathrm{Cu} /$ paper & Electroless deposition & $0.01 \Omega \mathrm{sq}^{-1}$ & 1 \\
\hline Ag NWs/paper & Dip-coating process & $0.51 \Omega \mathrm{sq}^{-1}$ & 2 \\
\hline RGO/PPy/paper & $\begin{array}{c}\text { In situ polymerization/chemical } \\
\text { reduction }\end{array}$ & $1.7 \Omega \mathrm{sq}^{-1}$ & 3 \\
\hline
\end{tabular}

\begin{tabular}{|c|c|c|c|}
\hline \multirow{3}{*}{ TOABr/Au/paper } & \multirow[t]{2}{*}{ ligand-mediated layer-by-layer } & \multirow{3}{*}{$0.3 \Omega \mathrm{sq}^{-1}$} & \multirow{3}{*}{4} \\
\hline & & & \\
\hline & assembly & & \\
\hline RGO/CNC paper & spin assisted layer-by-layer & $5000 \mathrm{~S} \mathrm{~m}^{-1}$ & 5 \\
\hline Ag NWs/CNC paper & Wax-printing + vacuum filtration & $3770 \mathrm{~S} \mathrm{~m}^{-1}$ & 6 \\
\hline CNT/CNC paper & Wax-printing + vacuum filtration & $115 \mathrm{~S} \mathrm{~m}^{-1}$ & 6 \\
\hline
\end{tabular}

Polyelectrlyte-induced solution-

$\mathrm{Cu} /$ cotton fabric

$1 \mathrm{~S} \mathrm{~m}^{-1}$

process

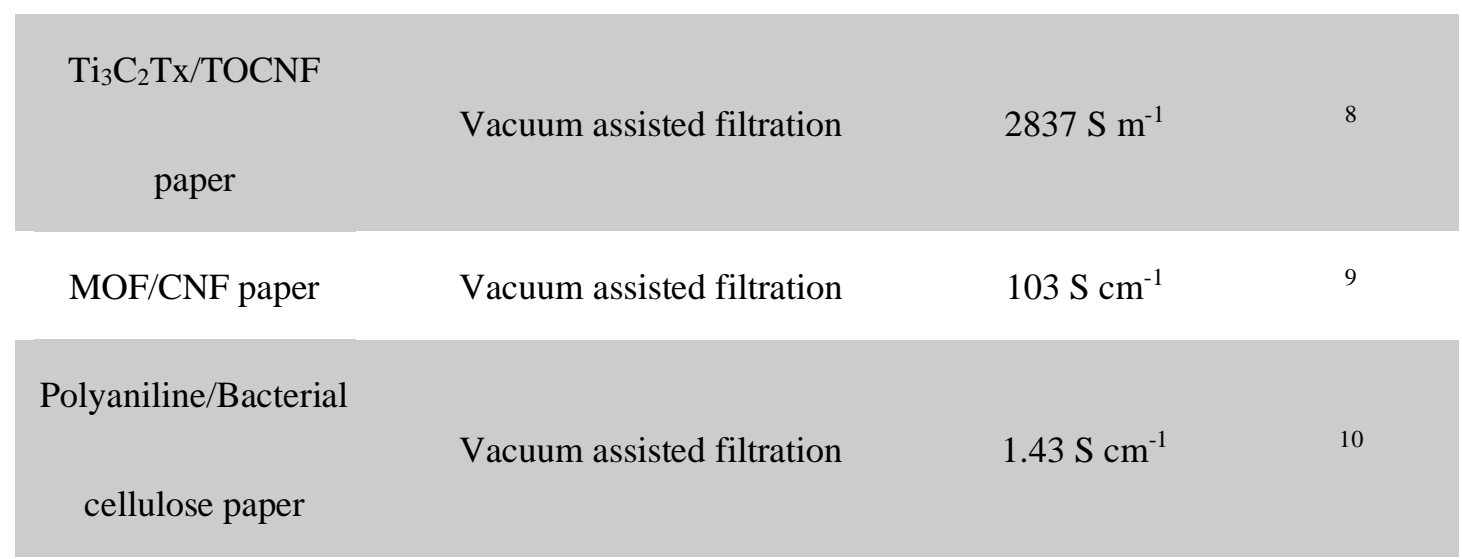

$\begin{array}{lc} & \text { Cycled film-liquid interface } \\ \text { Graphene/BC paper } & 101 \mathrm{~S} \mathrm{~cm}^{-1}\end{array}$


A organic linker-modulated ligand

$\mathrm{TOABr} / \mathrm{Au} / \mathrm{paper}$

exchange reaction and in situ

$2.2 \times 10^{5} \mathrm{~S} \mathrm{~m}^{-1}$

12

metallic fusion

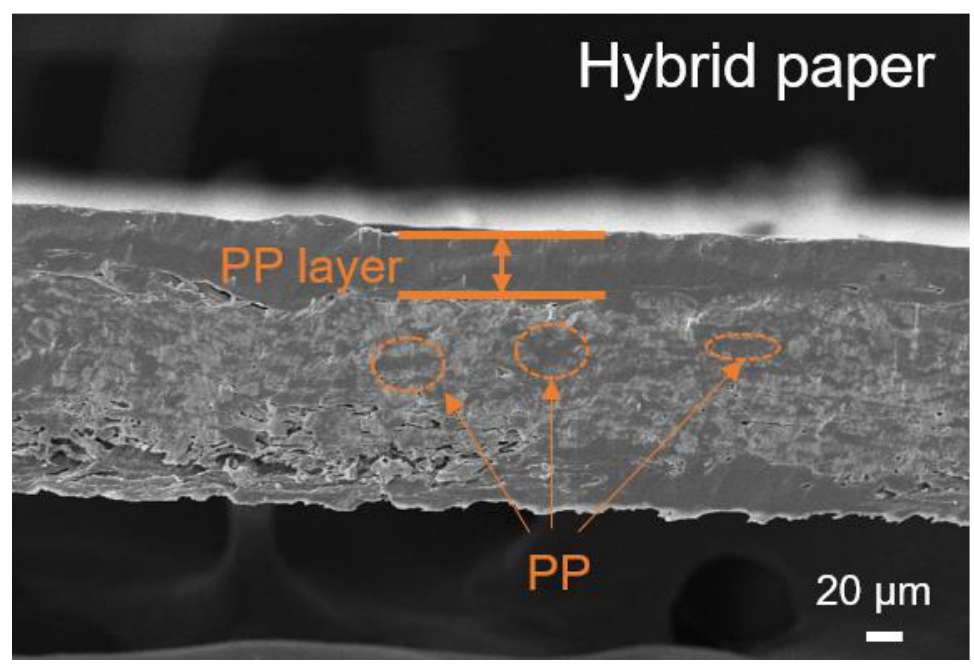

Figure S1. Cross-sectional SEM image of the hybrid paper.
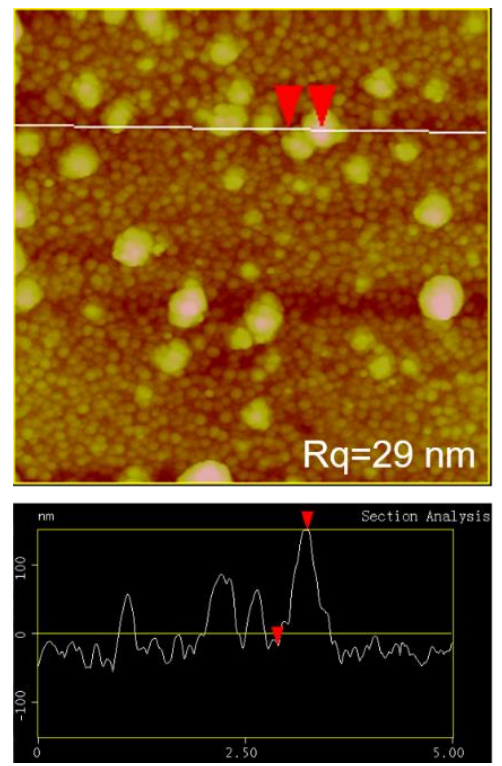

Figure S2. The AFM images and the height profiles of $\mathrm{Cu}$ NPs coated on HP with polydopamine as the adhesive layer. 


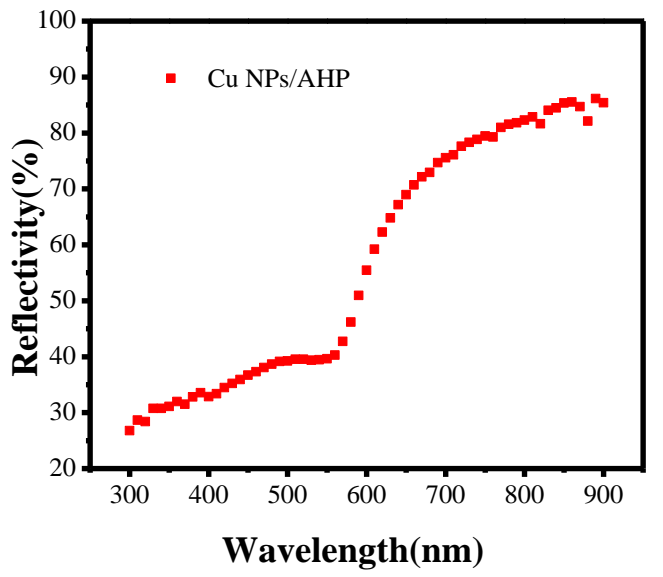

Figure S3. Reflectivity of the Cu NPs/AHP.
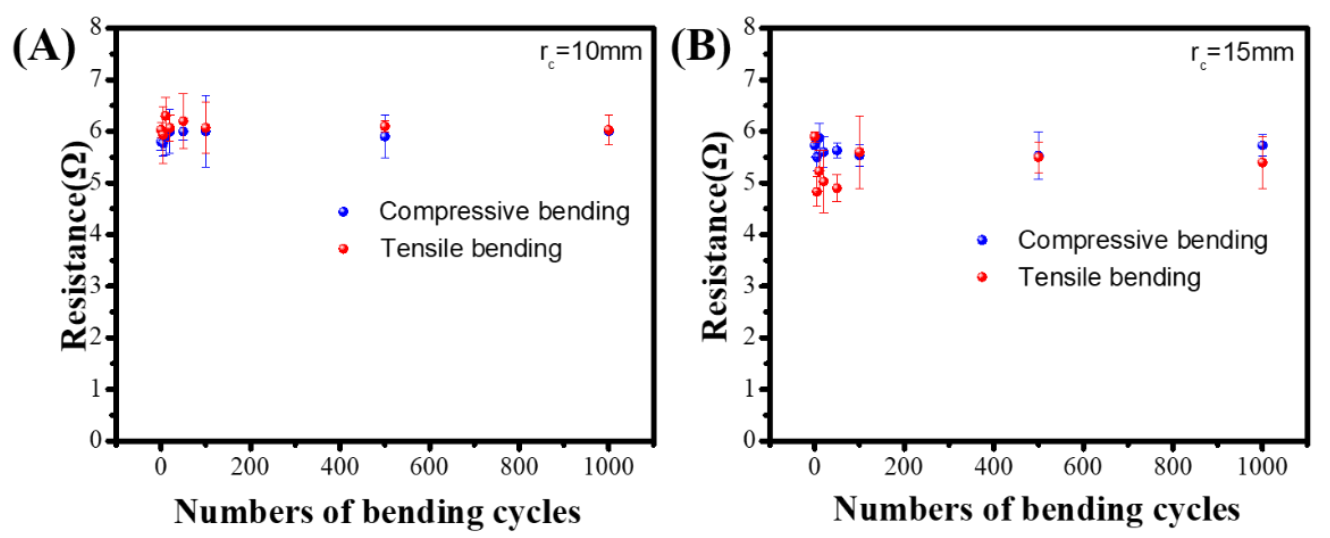

Figure S4. Resistance changes of the $\mathrm{Cu}$ NPs/AHP with different bending direction and cycles under curve radius of $10 \mathrm{~mm}$ (A) and $15 \mathrm{~mm}(\mathrm{~B})$.
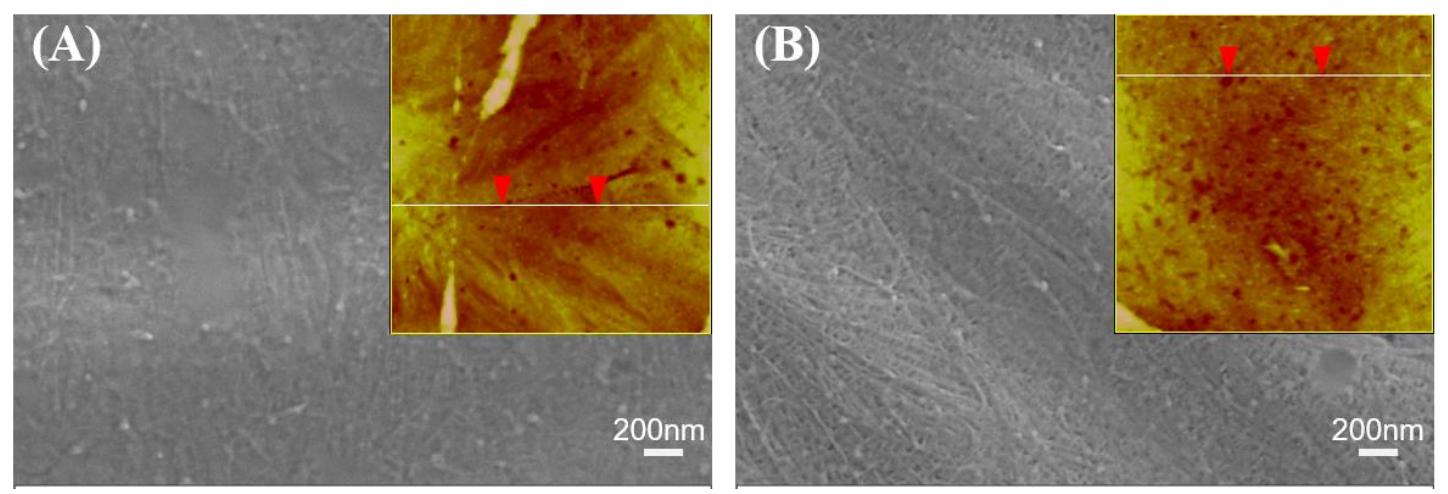

Figure S5. SEM and AFM images of hybrid paper before (A) and after acid-etched pretreatment (B). 


\section{References}

1. Yang, Y.; Huang, Q.; Payne, G. F.; Sun, R.; Wang, X., A highly conductive, pliable and foldable $\mathrm{Cu} /$ cellulose paper electrode enabled by controlled deposition of copper nanoparticles. Nanoscale 2019, 11 (2), 725-732, DOI 10.1039/C8NR07123C.

2. Li, J. P.; Wang, B.; Ge, Z.; Cheng, R.; Kang, L.; Zhou, X. M.; Zeng, J. S.; Xu, J.; Tian, X. J.; Gao, W. H.; Chen, K. F.; Qiu, C. Y.; Cheng, Z., Flexible and Hierarchical 3D Interconnected Silver Nanowires/Cellulosic Paper-Based Thermoelectric Sheets with Superior Electrical Conductivity and Ultrahigh Thermal Dispersion Capability. ACS Appl. Mater. Interfaces 2019, 11 (42), 39088-39099, DOI 10.1021/acsami.9b13675.

3. Wan, C. C.; Jiao, Y.; Li, J., Flexible, highly conductive, and free-standing reduced graphene oxide/polypyrrole/cellulose hybrid papers for supercapacitor electrodes. J. Mater. Chem. A 2017, 5 (8), 3819-3831, DOI 10.1039/C6TA04844G.

4. Ko, Y.; Kwon, M.; Bae, W. K.; Lee, B.; Lee, S. W.; Cho, J., Flexible supercapacitor electrodes based on real metal-like cellulose papers. Nat. Commun. 2017, 8, 536, DOI 10.1038/s41467-017-00550-3.

5. Xiong, R.; Hu, K. S.; Grant, A. M.; Ma, R. L.; Xu, W. N.; Lu, C. H.; Zhang, X. X.; Tsukruk, V. V., Ultrarobust Transparent Cellulose Nanocrystal-Graphene Membranes with High Electrical Conductivity. Adv. Mater. 2016, 28 (7), 15011509, DOI 10.1002/adma.201504438.

6. Hajian, A.; Wang, Z.; Berglund, L. A.; Hamedi, M. M., Cellulose Nanopaper with Monolithically Integrated Conductive Micropatterns. Adv. Electron. Mater. 2019, 5 (3), 1800924, DOI 10.1002/aelm.201800924 .

7. Liu, X.; Chang, H.; Li, Y.; Huck, W. T.; Zheng, Z., Polyelectrolyte-bridged metal/cotton hierarchical structures for highly durable conductive yarns. ACS Appl. Mater. Interfaces 2010, 2 (2), 529-535, DOI 10.1021/am900744n.

8. Zhan, Z. Y.; Song, Q. C.; Zhou, Z. H.; Lu, C. H., Ultrastrong and conductive MXene/cellulose nanofiber films enhanced by hierarchical nano-architecture and interfacial interaction for flexible electromagnetic interference shielding. J. Mater. Chem. C 2019, 7 (32), 9820-9829, DOI 10.1039/C9TC03309B.

9. Zhou, S. Y.; Kong, X. Y.; Zheng, B.; Huo, F. W.; Stromme, M.; Xu, C., Cellulose Nanofiber@ Conductive Metal-Organic Frameworks for High-Performance Flexible Supercapacitors. ACS Nano 2019, 13 (8), 9578-9586, DOI 10.1021/acsnano.9b04670.

10. Fei, G. Q.; Wang, Y.; Wang, H. H.; Ma, Y. N.; Guo, Q.; Huang, W. H.; Yang, D.; Shao, Y. M.; Ni, Y. H., Fabrication of Bacterial Cellulose/Polyaniline Nanocomposite Paper with Excellent Conductivity, Strength, and Flexibility. ACS Sustain. Chen. Eng. 2019, 7 (9), 8215-8225, DOI 10.1021/acssuschemeng.8b06306.

11. Luo, H. L.; Xie, J.; Xiong, L. L.; Zhu, Y.; Yang, Z. W.; Wan, Y. Z., Fabrication of flexible, ultra-strong, and highly conductive bacterial cellulose-based paper by engineering dispersion of graphene nanosheets. Composites Part B-Engineering 2019, 162, 484-490, DOI 10.1016/j.compositesb.2019.01.027. 
12. Kang, S.; Nam, D.; Choi, J.; Ko, J.; Kim, D.; Kwon, C. H.; Huh, J.; Cho, J., Highly Conductive Paper/Textile Electrodes Using Ligand Exchange ReactionInduced in Situ Metallic Fusion. ACS Appl. Mater. Interfaces 2019, 11 (12), 12032-12042, DOI 10.1021/acsami.8b21445. 